\title{
Implementation of MEMS Capacitive Pressure Sensor Design using COMSOL Software
}

\author{
Arunkumar Madupu, Sreenivasa Rao Ijjada, Gowri Ishwari
}

\begin{abstract}
This paper is based on the research of emerging implantable medical instrumentations. An implantable Blood Pressure (BP) monitoring Microsystems is very essential to monitor patient's BP continuously and gives warning when they are extreme and interim levels. The instrument should be accurately measure the both systolic and diastolic values. Based on the readings the $B P$ is categorized in to four (Ideal BP: diastolic $=120$ and systolic $=80$, low $\mathrm{BP} 90$ and 60, Pre-hyper values are 140 and 90 and hyper tension values are 190 and 100). BP consists one of the principle vital signs referring to the force entered by circulating blood on the walls of blood vessels. The system consists of instrumented elastic cuff, wrapped around a blood vessel, to sense real time blood pressure. In this paper, a MEMS based capacitor pressure sensor is to be designed with less than the dimensions of $0.4 \times 0.5 \times 0.4 \mathrm{~mm}^{3}$, and with the approximate capacitance of less than $2 \mathrm{pF}$ and $1 \mathrm{FF}$ per $\mathrm{mmHg}$.
\end{abstract}

Keywords: MEMS, capacitive sensor, Blood Pressures, COMSOL software.

\section{INTRODUCTION}

Sensor collects the information in the analog domain, amplified and then links the collected information to the analog to the digital converters for signal conditioning. Sensors collects the data in different physical from the environment are light, pressure, motion, temperature, etc. Sensors are integrated with actuators. A motor is responsible for moving or controlling a mechanism or system is an actuator [13]. Actuator is operated with a source of energy, typically electric current, hydraulic fluid pressure and converts that energy into the motion. Signal conditioning is the unit which is used to filter, amplify or modify the output of the sensor to get a desired output voltage. A Sensor's sensitivity indicates how much the sensor's output changes when the input quantity being measured changes [14]. The sensor block diagram is shown in the fig.1. There are two basic sensing principles as i) Piezoresistive sensing principle and ii) capacitive sensing principle.

\section{i) Piezoresistive sensor:}

The prefix "Piezo" means 'squeeze' or 'press. The piezoresistive sensing principle uses electric effect to measure the mechanical stress/ electric charge in the doped resistor area in terms pressure, acceleration, temperature, strain, or force. The physical quantities are converting into an electrical charge.

Revised Version Manuscript Received on August 19, 2019.

Arunkumar Madupu, Research Scholar, Dept. of ECE, GIT, GITAM (Deemed to be University), Visakhapatnam,A.P., India.

Sreenivasa Rao Ijjada Senior Member IEEE, Department of Electronics and Communication Engineering, GITAM University, Visakhapatnam, A.P., India

Dr.Gowri Ishwari, Dental Surgeon, Sri shobha Multispecialty dental clinic and implant center, Visakhapatnam,A.P, India.

Applications are i) load cell, ii) accelerometer and iii) pressure sensor. Piezoresistive sensor works in three operational modes as transverse, longitudinal and shear effect.

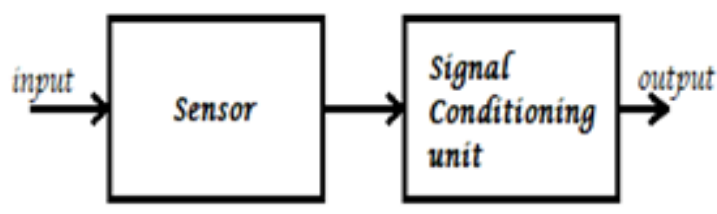

Fig. 1: Block diagram of Sensor

ii)Capacitive Pressure sensor:

This principle uses to measure the deflection, which can be resulted due to the change in distance between the capacitor plates.

\section{MEMS Technology:}

The micro-electromechanical system (MEMS) is a technology used to generate micro-miniature electronic mechanical device. The MEMS pressure sensor is a sensing device that can measure and detect the external incentives such as pressure, and then it can response to the measured pressure. MEMS (Micro-Electromechanical Systems) are of light weight, small size, reliable systems and enhanced performance. MEMS pressure sensors quota the pressure in terms of deflection of sensing plate. Micro pressure sensors are extensively applied in biomedical, automotive, military, space, and numerous industrial applications. MEMS technology fabricates pressure sensors and has the profits of low cost, small size and high performance. The material used to fabricate the MEMS Pressure sensor is micro sized. These devices are developed by manufacturing and fabrication at micro level.

\section{CLASSIFICATION OF PRESSURE SENSORS}

MEMS are trivial micro mechanized systems that normally are used for various purposes enclosed in small chips [1]. MEMS technology clubs silicon based micromachining technology and microelectronics. Pressure sensors are required in many applications like defense, biomedical, automobile and many more civilian \& domestic applications. Original pressure sensors were established using strain gauge mechanism but nowadays there is a rapid development in packaging and fabrication abilities. MEMS pressure sensors miniaturization and design transformed after outcome of Piezo-resistivity in germanium and silicon [2-3]. MEMS are 
used to grow micro scale biosensors that will be useful for many biological operations. It is also used for various commercial and industrial applications. Micro cantilevers have ability to show higher sensitivity [3-4].

\section{Capacitive Pressure Sensors:}

This type of pressure sensor makes parallel plate capacitive transduction principle usage. The applied pressure creates capacitance change between two plates. This uses diaphragm as one movable electrode with respect to fixed electrode. The charge produces a potential difference which may be sustained using external voltage. A capacitive pressure sensor measures pressure by noticing an electrostatic capacitance change. The deflection of the diaphragm results to the change in capacitance, which can be readout as electrical signal by using suitable mechanisms like Capacitance Bridge [6-8]. Capacitive sensors have the advantage over the piezoresistive type, that they consume less power, invariant of temperature but have a nonlinear output signal [9]. Capacitive sensors are well-suited with most mechanical structures, and they have low temperature drift and high sensitivity.

\section{Piezoelectric Pressure Sensors:}

Barium titanate, single-crystal quartz, leads (PZT) etc., can generate an electrical signal and a potential difference when they are subjected to mechanical stress or strain [10]. When pressure is applied to a material it creates a strain or deformation in the material [11]. In a piezoelectric material strain generates an electrical voltage or potential difference.

\section{Resonant Pressure Sensors:}

The pressure measurement in this is built on the resonant frequency of structures. Here due to external pressure applied there will be change in resonant frequency of structure and that change is an mark of pressure $[5,12]$. Resonant pressure sensors are widely used nowadays because of that measurement of frequency is one of the high precision and robust methods existing.

Utmost people are suffering from (hypertension) high blood pressure. Some of these patients can hardly be calmed on drugs and for some of this group a long term monitoring is advisable. Till now conservative extracorporeal systems like blood pressure cuffs for long-term monitoring with specific measurements are used which tend to be a handicap for affected patients due to their size especially at night. Furthermore those cuffs do not allow continuous measurements. For aggressive blood pressure monitoring catheters are existing for short-term use during surgery. These are restricted to clinical evaluation due to the essential everlasting access to the body.

\section{DESIGN OF CAPACITIVE PRESSURE SENSOR}

The designing of MEMS Capacitive Pressure Sensor is carried out in COMSOL Multi physics Software. Flowchart for the implantation of MEMS capacitive Pressure sensor in COMSOL Software is as shown in figure 2. The design created in COMSOL is as shown in figure 3.

The flowchart shown in the figure 2 represents the step by step procedure for the design of the capacitive MEMS sensor using COMSOL software.

\section{Material properties:}

The material properties used in this design are listed in the below table: The total design is divided into four quadrants, one of the quadrants is as shown in figure 4.

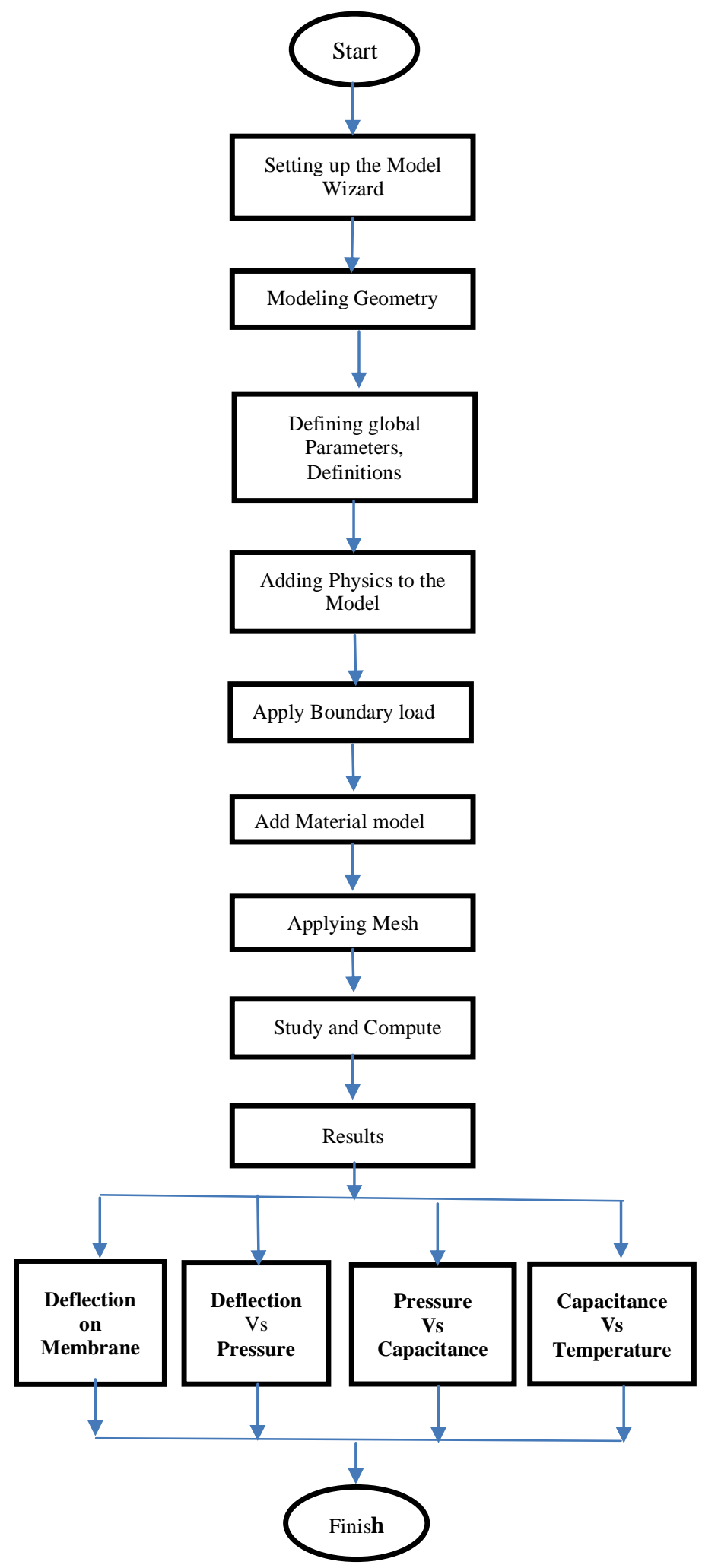

Figure 2: Flowchart of COMSOL implementation

Published By:

Blue Eyes Intelligence Engineering 


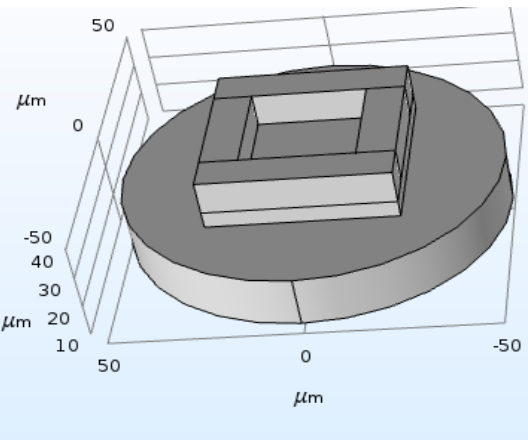

Figure 3: Design of Capacitive pressure sensor in COMSOL

Table1: Properties of material used in design

\begin{tabular}{|c|c|c|c|}
\hline NAME & $\mathbf{S i}$ & $\mathrm{Si}^{\text {Poly- }}$ & $\begin{array}{l}\text { Diamo } \\
\text { nd }\end{array}$ \\
\hline Density $\left(\mathrm{kg} / \mathrm{m}^{3}\right)$ & 29 & 2320 & 3520 \\
\hline $\begin{array}{l}\text { Youngs } \\
\text { Modulus (Gpa) }\end{array}$ & $0^{16}$ & 169 & 1050 \\
\hline Poisson's ratio & $8^{0.2}$ & 0.22 & 0.2 \\
\hline
\end{tabular}

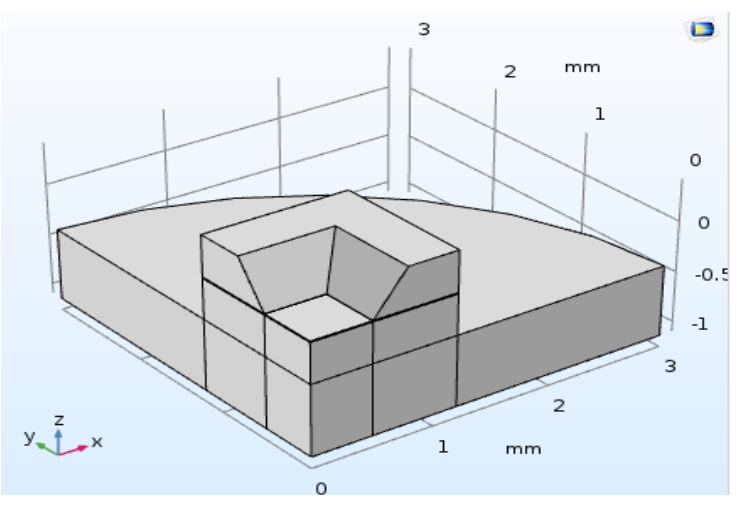

Figure 4: Quarter part of deigned pressure sensor

Silicon, Polymers, Metals, Ceramics are used. Silicon and silicon compounds are used for designing the capacitive pressure sensor. Silicon is widely used because it can be processed with bulk micro-machining. Silicon is used because it is a high insulating material. Silicon has low dielectric losses. Polymer materials are used because these materials are more flexible compared to that of silicon. The displacement of capacitive sensor is shown in the figure 5 when the pressure is applied.

\section{Displacement (emi)}

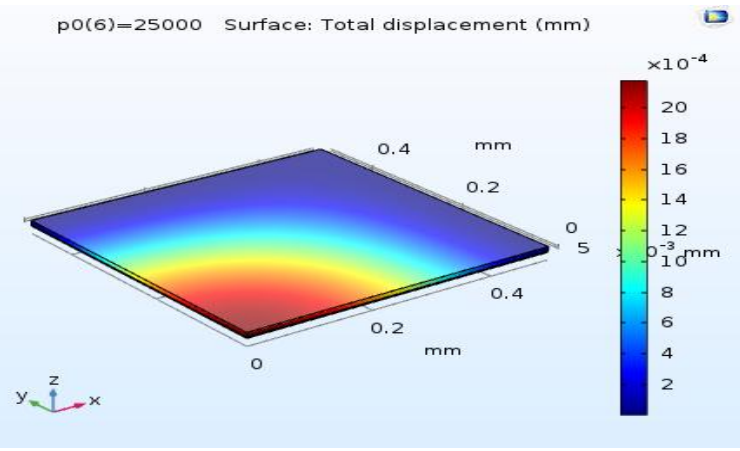

Figure 5: Displacement of the membrane

\section{RESULTS}

The results of capacitive pressure sensor to measure the blood pressure are shown in the figures from figure 6-figure 17.

\section{i) Diaphragm Displacement versus Pressure}

The maximum displacement of the membrane is now non-zero at zero applied pressure, as a result of the packaging stress. The displacement-pressure line gradient also has changed. The figure 6 shows the diaphragm displacement with respect to the applied pressure, from this it is observed that the zero pressure displacement is maximum.

\begin{tabular}{|l|l|l|}
\hline Expression & Unit & Description \\
\hline emi.C11 & Pf & $\begin{array}{l}\text { Capacitance with } \\
\text { Package stress }\end{array}$ \\
\hline
\end{tabular}

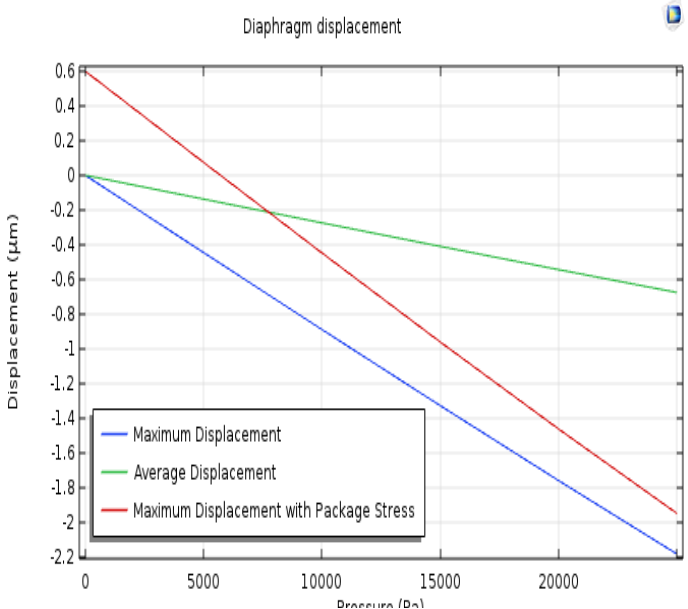

Figure 6: Diaphragm Displacement vs Pressure

ii) Model Capacitance versus Pressure:

the capacitance of the capacitive pressure is modeled with the pressure. When the pressure is applied, the displacement of the membrane takes place, there by the capacitance varies as shown in the figure 7.

Model Capacitance vs Pressure

(

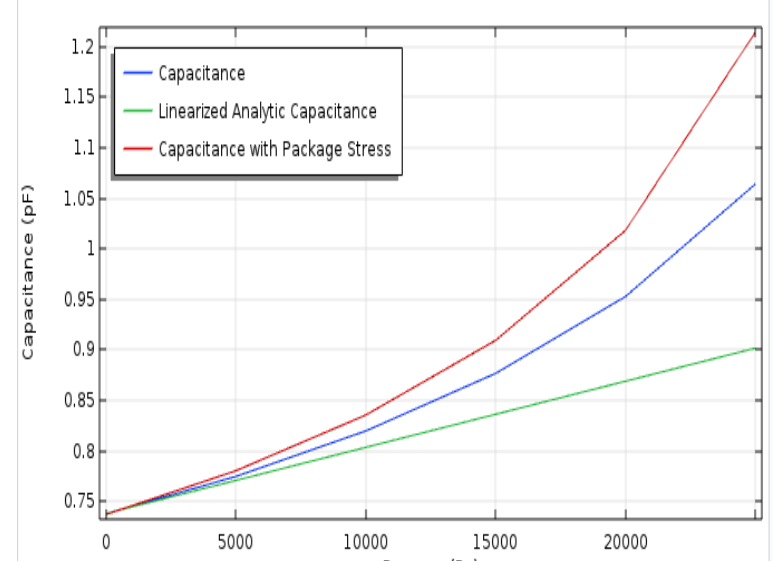

Fig7: Model Capacitance versus Pressure 


\section{iii) Capacitance versus Operating Temperature:}

The capacitance of the capacitor strongly depends on the temperature at which it operates. The figure 8 shows that the variation of capacitance with respect to the temperature.

Model Capacitance vs Operating Temperature

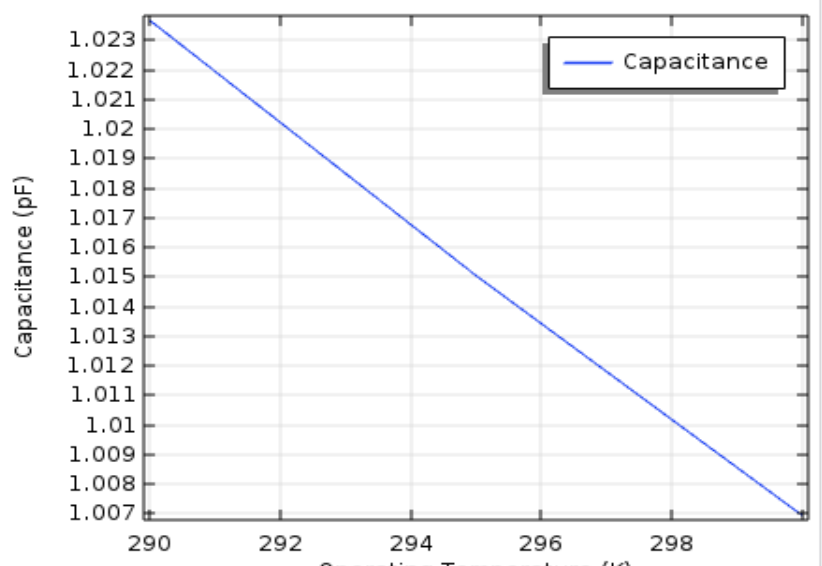

Fig8: Capacitance versus Operating Temperature

The above Results are obtained by considering diaphragm material as Silicon. Now the material of the diaphragm is changed as Silicon [solid, <111>axis] and observe the changes that are obtained. The capacitance decreases with increase in Temperature. There is a decrease in capacitance of 0.078 when compared to the above graph that is by considering silicon material. The displacement versus pressure is shown in the figure 9. And the capacitance versus pressure is shown in the figure 10.

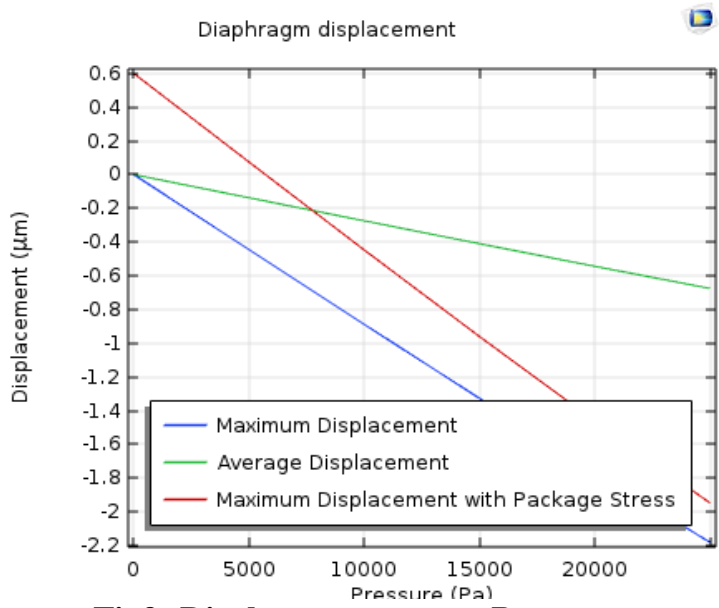

Fig9: Displacement versus Pressure

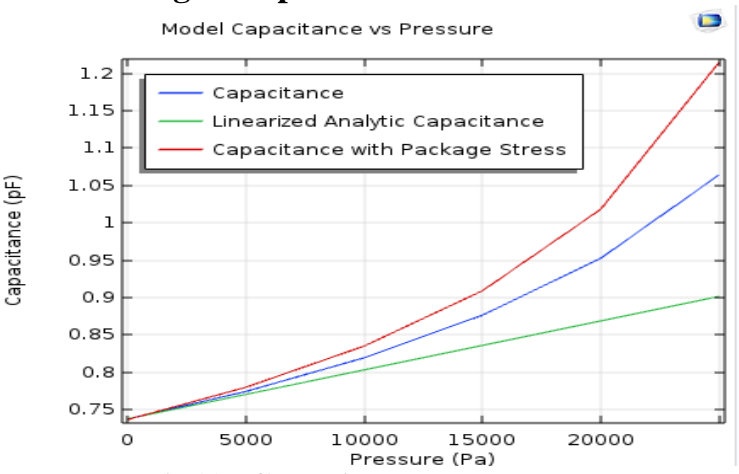

Fig10: Capacitance versus Pressure

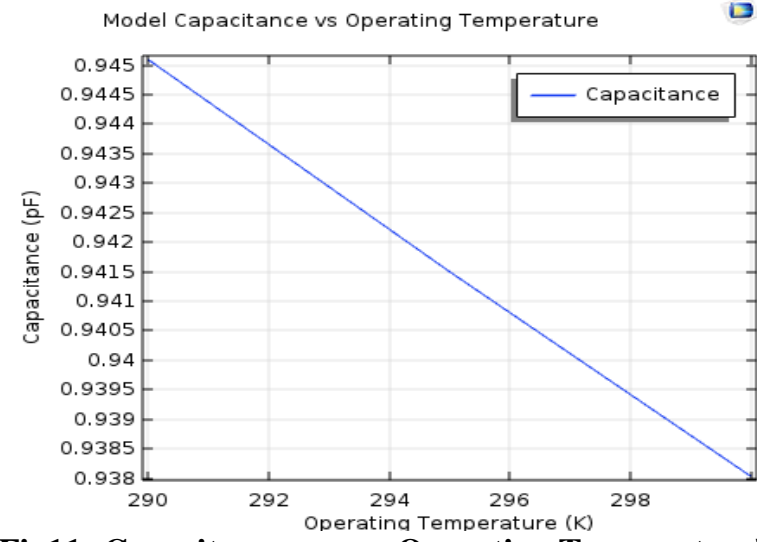

Fig11: Capacitance versus Operating Temperaturel

By change the material of the diaphragm as Silicon [solid, $<110>$ axis] and observe the changes that are obtained. The figure 12 to figure 14 shows the displacement vs pressure, capacitance vs pressure and capacitance vs temperature.

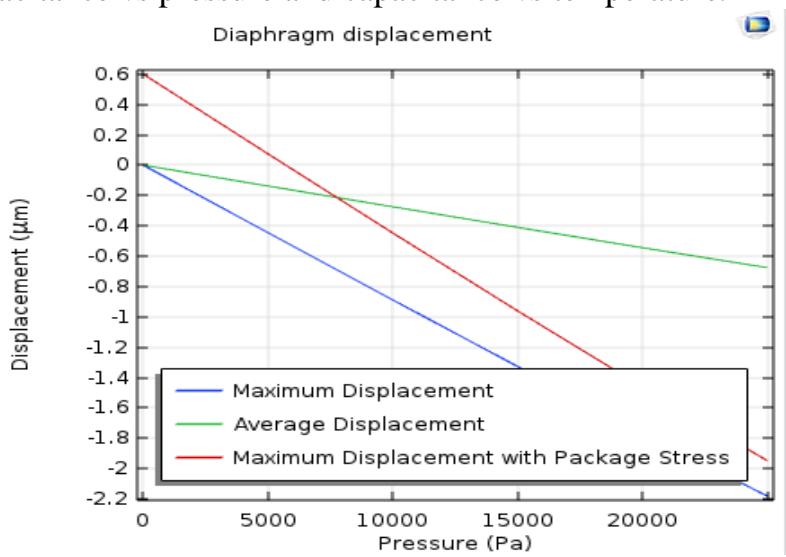

Fig12: Displacement versus Pressure

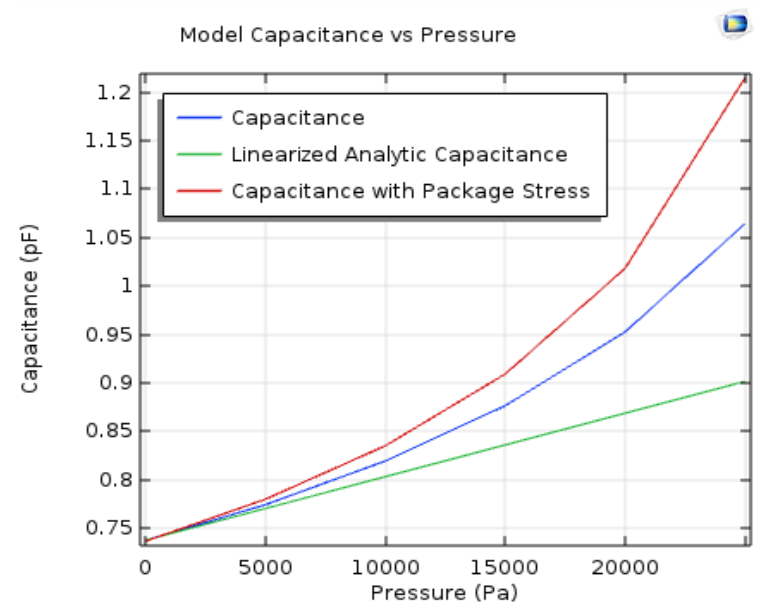

Fig13: Capacitance versus Pressure 


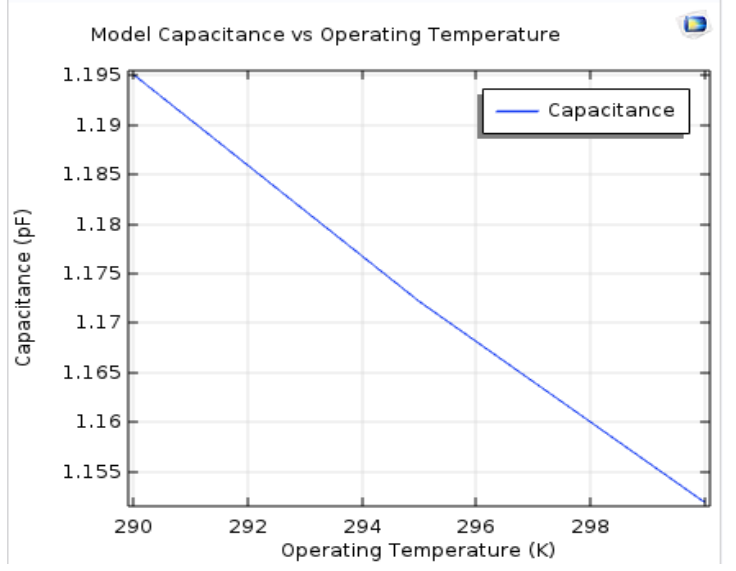

Fig14: Capacitance versus Operating Temperature

The Capacitance increases 0.25 at the same temperature when compared to Silicon [solid, <111>axis] material. The figure 15 to figure 17 shows the displacement vs pressure, capacitance vs pressure and capacitance vs temperature.

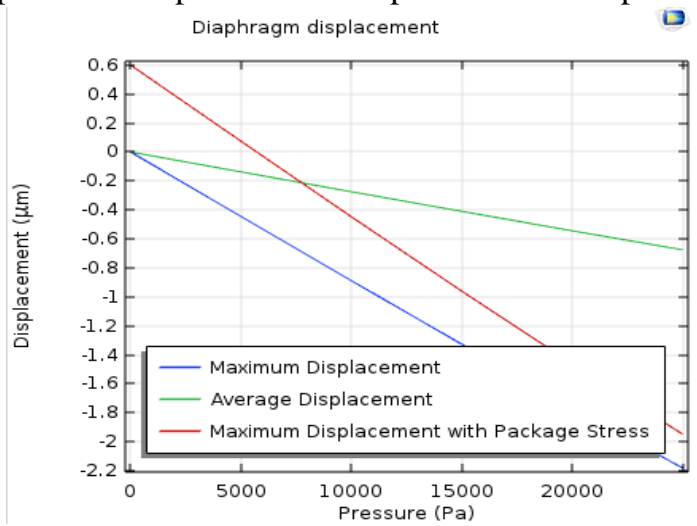

Fig15: Displacement versus Pressure

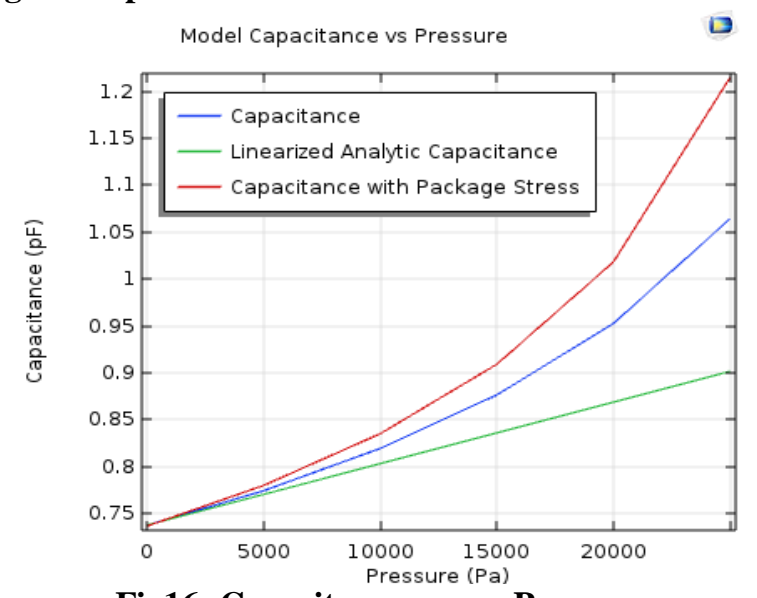

Fig16: Capacitance versus Pressure

Model Capacitance vs Operating Temperature

Q

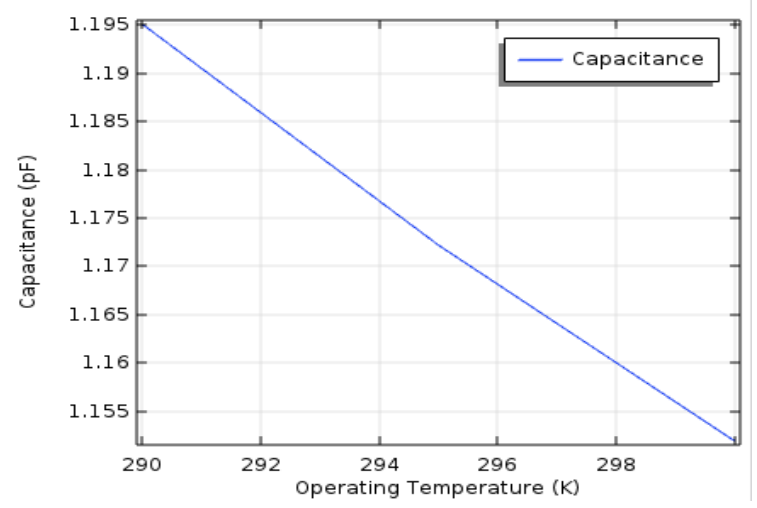

Fig17: Capacitance versus Operating Temperature
The capacitance remains same when compared to Silicon [solid, <110>axis] at same temperature. The capacitance increases 0.25 at same temperature when compared to Silicon [solid, <111>axis]. The capacitance increases 0.172 at same temperature when compared to Silicon.

\section{CONCLUSION}

The MEMS Capacitive pressure prototype has been studied and designed. The results obtained from designed prototype the relation between the applied pressure and the capacitance, we can conclude from that the capacitive pressure is highly sensitive. We can also observe change in capacitance with variation in operating temperature. Since the variations are very slight, the capacitive sensor is low temperature dependence. The capacitor has low power consumptions as we applied low voltage between the parallel plates.

\section{ACKNOWLEDGEMENT}

We would like to thank Department of Electronics and communications engineering, GIT, GITAM deemed to be University Faculty and staff for supporting to prove software and lab facilities.

\section{REFERENCES}

1. D. Klaitabtim and A. Tuantranont, "Design consideration and finite element modeling of MEMS cantilever for nanobiosensor applications", Procs. of 2005 5th IEEE Conference on Nanotechnology, Nagoya, Japan, July 2005.

2. C. S. Smith, Piezoresistance effect in germanium and silicon, Physical Review, 94, 1, 1954, pp. 42-49.

3. Tufte O. N., Chapman P. W., Long D., Silicon diffused-element piezoresistive diaphragms, J Appl. Phys., 33, 1962, pp. 3322-3327.

4. Jeff Melzak, Nelsimar Vandelli, SiC MEMS Pressure Sensors: Technology, Applications and Markets, PLXmicro.

5. K. N. Bhat, M. M. Nayak, MEMS Pressure Sensor, An overview of challenges in 'Technology and Packaging', Journal of ISSS, Vol. 2, No. 1, March 2013, pp. 39-71.

6. C. S. Sander, J. W. Knutti, J. D. Meindle, A monolithic capacitive pressure sensor with pulse period output, IEEE Transactions on Electron Devices, 1980, pp. 927-930.

7. C. S. Sander, J. W. Knutti, J. D. Meindl, A monolithic capacitive pressure sensor with pulse-period output, IEEE Transactions on Electron Devices, Vol. 27, Issue 5, 1980, pp. 927-930.

8. Gitesh Mishra, Neha Paras, Arti Arora, P. J. George, Simulation of MEMS Capacitive Pressure Sensor Using Comsol Multhysics, International Journal of Applied Engineering Research, Vol. 7, No. 11, 2012.

9. Tamas Karpati, Andrea Edit Pap, Sandor Kulinyi, Prototype MEMS Capacitive Pressure Sensor Design and Manufacturing, Electrical Engineering and Computer Science, 57, 1, 2013, pp. 3-7.

10. Stanley Kon, Roberto Horowitz, High-resolution MEMS, IEEE Sensors Journal, Vol. 8, No 12, December 2008, pp. 2027-2035

11. Stanley Kon, Kenn Oldham, Roberto Horowitz, Piezoresistive and Piezoelectric MEMS Strain Sensors for Vibration Detection, Sensors and Smart Structures Technologies for Civil, Mechanical and Aeorospace Systems, Proc. SPIE, 6529, 2007.

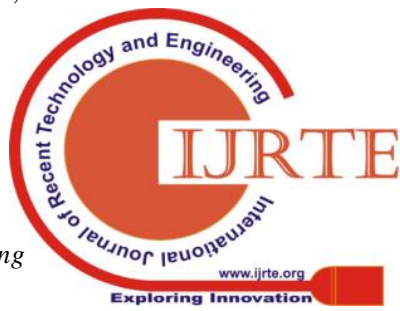


12. Martin A. Schmidt, Roger T. Howe, Silicon Resonant Microsensors, in Proceedings of the 14th Automotive Materials Conference: Ceramic Engineering and Science Proceedings, Vol. 8, Issue 9-10, September. October 1987, Chapter 3.

13. Peng Cong, "A Wireless and Baterryless 10 bit implantable blood pressure sensing microsystem with adaptive RF powering for real time laboratory mice monitoring", IEEE Journal of Solid State Circuits.

14. SMARTEC "About Pressure Sensor", pp.1-7, The Netherlands. 